\title{
Comparison of Cognitive Function of Ischemic Stroke Patients With and Without Pneumonia
}

\author{
Amanda Diannisa Azzahra, ${ }^{1}$ Lisda Amalia, ${ }^{2}$ Chandra Calista, ${ }^{2}$ Sofiati Dian, ${ }^{2}$ Siti Aminah, ${ }^{2}$ \\ Paulus Anam Ong ${ }^{2}$ \\ ${ }^{1}$ Faculty of Medicine Universitas Padjadjaran, Indonesia, ${ }^{2}$ Departement of Neurology Faculty of \\ Medicine Universitas Padjadjaran/Dr. Hasan Sadikin General Hospital, Bandung, Indonesia
}

\section{Abstract}

Background: Pneumonia is a common complication of ischemic stroke that may worsens brain injury, leading to cognitive impairment as well as patient outcome. The aim of this study was to compare cognitive function in ischemic stroke patients with and without pneumonia.

Methods: This study was a comparative numerical analytic retrospective cross-sectional study that was conducted from September 2020 to February 2021. This study used data from ischemic stroke patients treated at Dr. Hasan Sadikin General Hospital in 2019 with the total sampling method. Data were stratified into patients with pneumonia and without pneumonia. The mini mental state examination (MMSE) was used to measure cognitive function in both groups and scores were compared using the Mann-Whitney Test.

Results: Of the 164 data, 25 were patients with pneumonia and 139 without pneumonia. Male patients were predominantly prevalent in the pneumonia and non-pneumonia groups. Hemiparesis was the most common neurological deficit. The MMSE score was significantly lower in the pneumonia group (22.6 \pm 5.4 vs. $24.7 \pm 4.8$, respectively; $p$-value $=0.032$ ).

Conclusions: Cognitive function is more prevalent in ischemic stroke patient with pneumonia compared to non-pneumonia. However, other factors such as recurrent stroke and possible vascular risk factors should be considered for future studies to better identify the relationship between ischemic stroke and pneumonia.

Keywords: Cognitive function, ischemic stroke, pneumonia

\section{Introduction}

Stroke is the most common cause of disability in adults. Neurologic deficit after stroke, such as neurologic deterioration, neuromuscular, psychiatry, and cognitive impairment are the causes of disability. ${ }^{1}$ About $25-30 \%$ of stroke survivors develop vascular cognitive impairment (VCI) or vascular dementia (VaD). ${ }^{2}$ Neuroanatomicallesion, cerebralvesselslesion, and Alzheimer's disease contribute to poststroke cognitive impairment. ${ }^{3}$ Pneumonia is a common complication following acute ischemic stroke with a reported incidence of 5-26\%. ${ }^{4}$ The incidence of pneumonia correlates with prolonged immobilization, decreased level of consciousness, and dysphagia. ${ }^{5}$ Pneumonia, once it occurs, activates an adaptive immune response directed against Central Nervous System (CNS) antigens thus worsening brain injury after stroke. ${ }^{6}$ Patients hospitalized with pneumonia only were associated with worse functional and cognitive impairment. ${ }^{7}$ The possibility of pneumonia complication may worsen the cognitive function of ischemic stroke patients.

The mini mental state examination (MMSE) is widely used as a screening tool for cognitive deficits. This includes tests of orientation, attention, memory, language, and visuospatial skills. ${ }^{8}$ This instrument consists of 11 questions that only take 5-10 minutes to complete. ${ }^{7}$ The objective of this study was to compare cognitive function in ischemic stroke patients with and without pneumonia.

Correspondence: Amanda Diannisa Azzahra, Faculty of Medicine, Universitas Padjadjaran, Jalan Raya Bandung Sumedang Km. 21 Jatinangor, Sumedang, Indonesia, E-mail: amanda17001@mail.unpad.ac.id 


\section{Methods}

This observational analytic comparative numerical study used secondary data from medical records of ischemic stroke patients who were admitted to Dr. Hasan Sadikin General Hospital Bandung. The study was conducted from September 2020 to February 2021. Measurement of cognitive function was assessed using the MMSE.

Medical records of ischemic stroke patients were collected from January to December 2019 which met the MMSE inclusion criteria with a total sampling technique. Medical records were excluded from this study when they had incomplete MMSE scores. The MMSE was not performed might be due to low level of education, decreased level of consciousness, and global or motoric aphasia and deceased. Furthermore, the level of consciousness was assessed by using the Glasgow Coma Scale (GCS) and Stroke deficit was assessed using National Institutes of Health Stroke Scale (NIHSS). This study has been approved by the Research Ethics Committee of Universitas Padjadjaran no. 025/UN6.KEP/EC/2021.

The data were tested for normality using the Shapiro-Wilk test for dataset of $<50$ samples. The Mann-Whitney test was used for the analysis of abnormally distributed continuous data. The analysis was performed by comparing the MMSE scores in the pneumonia and non-pneumonia groups. Statistically significant was considered when $\mathrm{p} \leq 0.05$. Data were analyzed using IBM $®$ SPSS ver. 25 .

\section{Results}

During the study period, 547 ischemic stroke patients were hospitalized at Dr. Hasan Sadikin General Hospital Bandung, however, only 164 were eligible for further analysis. The excluded subjects were 120 who were deceased and 263 without MMSE scores (153 with incomplete medical records data, 56 with severe aphasia, and 54 with decreased level of consciousness) as shown in Figure 1.

There were 164 eligible subjects consisting of 25 patients with pneumonia and 139 without pneumonia. Male patients were more predominantly prevalent in both pneumonia and non-pneumonia group (64\% and $56.1 \%$ respectively). The average of age in both subjects was not significantly different $(60 \pm 11.3$ in the pneumonia vs. $60.6 \pm 11$ in the non-pneumonia group). Education was slightly lower in the pneumonia group $(9.4 \pm 0.6$ vs. $9.7 \pm 0.2$ years of education). In the pneumonia group, most of the subjects were office worker $(44 \%)$ while subjects who were unemployed had the highest prevalence in the non-pneumonia group (49.6\%), as shown in Table 1.

The level of consciousness assessed using the Glasgow Coma Scale (GCS) tended to be lower in the pneumonia group than in the non-penumonia group $(14.2 \pm 1.8$ vs. $14.7 \pm 0.9$, respectively), although the scores were not statistically different. Stroke deficits assessed using the National Institutes of Health Stroke Scale (NIHSS) were higher in the pneumonia group $(8.5 \pm 3.7$ vs. $6.7 \pm 3.6$, respectively;

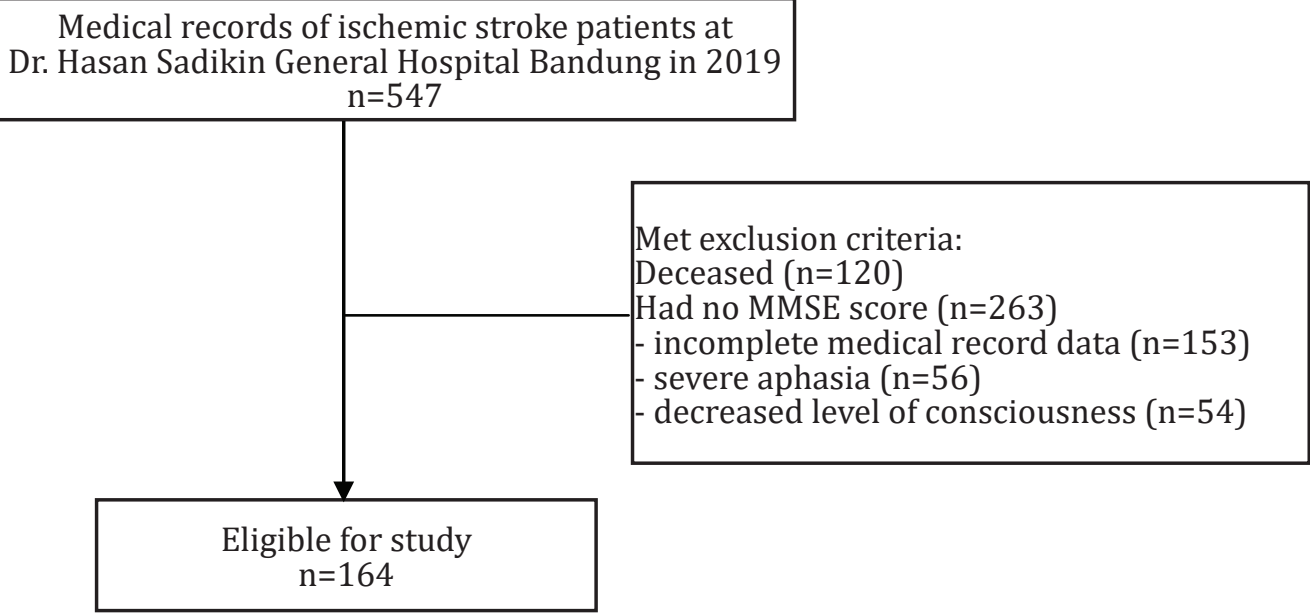

Figure 1 Subject Selection 
Table 1 Comparison of Demographic Characteristics of Ischemic Stroke Patients With or Without Pneumonia at Dr. Hasan Sadikin General Hospital in 2019

\begin{tabular}{lccc}
\hline \multicolumn{1}{c}{ Patients Characteristics } & $\begin{array}{c}\text { Pneumonia }(\mathbf{n}=\mathbf{2 5}) \\
\mathbf{n ( \% )}\end{array}$ & $\begin{array}{c}\text { No Pneumonia (n=139) } \\
\mathbf{n ( \% )}\end{array}$ & p-value \\
\hline Gender, n (\%) & & & \\
Men & $16(64)$ & $78(56.1)$ & 0.463 \\
Women & $9(36)$ & $61(43.9)$ & \\
Age & & & \\
Mean \pm SD & $60 \pm 11.3$ & $60.6 \pm 11$ & 0.889 \\
Median & 59 & 60 & \\
IQR & 8 & 14 & \\
Years of Education & & & \\
Mean \pm SD & $9.4 \pm 0.6$ & $9.7 \pm 0.2$ & \\
Median & 12 & 12 & \\
IQR & 6 & 6 & \\
Occupation, n (\%) & & & \\
Unemployed & $8(32)$ & $69(49.6)$ & \\
Office worker & $11(44)$ & $51(36.7)$ & \\
Entrepreneur & $4(16)$ & $5(3.6)$ & \\
Farmer/fisherman/laborer & $0(0)$ & $10(7.2)$ & \\
Others & $2(8)$ & $4(2.9)$ & \\
\hline
\end{tabular}

p-value 0.046). Hemiparesis was the most common neurologic deficitseen in both groups. Subjects with lesion in parietal lobe had the highest prevalence in pneumonia group
(24\%) while lesion in the basal ganglia had the highest prevalence in the non-pneumonia group (30.6\%). In the pneumonia group, most of the lesions were located on parietal lobe

Table 2 Clinical Characteristic of Ischemic Stroke Patients With and Without Pneumonia from Dr. Hasan Sadikin General Hospital in 2019

\begin{tabular}{|c|c|c|c|}
\hline Variable & $\begin{array}{c}\text { Pneumonia }(n=25) \\
n(\%)\end{array}$ & $\begin{array}{c}\text { No Pneumonia }(n=139) \\
n(\%)\end{array}$ & P-value \\
\hline $\begin{array}{l}\text { Level of consciousness (GCS) } \\
\text { Mean } \pm \text { SD } \\
\text { Median } \\
\text { IQR }\end{array}$ & $\begin{array}{c}14.2 \pm 1.8 \\
15 \\
0\end{array}$ & $\begin{array}{c}14.7 \pm 0.9 \\
15 \\
0\end{array}$ & 0.266 \\
\hline $\begin{array}{l}\text { Stroke Severity Level (NIHSS) } \\
\text { Mean } \pm \text { SD } \\
\text { Median } \\
\text { IQR }\end{array}$ & $\begin{array}{c}8.5 \pm 3.7 \\
7 \\
6.75\end{array}$ & $\begin{array}{c}6.7 \pm 3.6 \\
6 \\
5\end{array}$ & $0.046^{*}$ \\
\hline $\begin{array}{l}\text { Neurologic Deficit, n (\%) } \\
\text { Dysphagia } \\
\text { Dysarthria } \\
\text { Hemiparesis }\end{array}$ & $\begin{array}{l}7(28) \\
10(40) \\
21(84)\end{array}$ & $\begin{array}{c}16(11.5) \\
77(55.4) \\
122(87.8)\end{array}$ & 0.175 \\
\hline $\begin{array}{l}\text { Lesion Location, n (\%) } \\
\text { Frontal lobe } \\
\text { Parietal lobe } \\
\text { Temporal lobe } \\
\text { Occipital lobe } \\
\text { Cerebellum } \\
\text { Pons } \\
\text { Basal ganglia } \\
\text { Thalamus } \\
\text { Lateral periventricle }\end{array}$ & $\begin{array}{c}3(12) \\
6(24) \\
3(12) \\
1(4) \\
1(4) \\
1(4) \\
2(8) \\
0(0) \\
5(20)\end{array}$ & $\begin{array}{c}9(6.5) \\
19(13.7) \\
16(11.5) \\
11(7.9) \\
4(2.9) \\
5(3.6) \\
43(30.9) \\
8(5.8) \\
31(22.3)\end{array}$ & 0.348 \\
\hline
\end{tabular}


Table 3 Comparison of MMSE Scores in Ischemic Stroke Patients with and Without Pneumonia Complication

\begin{tabular}{lccc}
\hline \multicolumn{1}{c}{ Patients Characteristics } & $\begin{array}{c}\text { Pneumonia } \\
(\mathbf{n}=\mathbf{2 5})\end{array}$ & $\begin{array}{c}\text { No Pneumonia } \\
(\mathbf{n = 1 3 9 )}\end{array}$ & P-value \\
\hline Mean \pm SD & $22.6 \pm 5.4$ & $24.7 \pm 4.8$ & $0.032^{*}$ \\
Median & 23 & 26 & \\
\hline
\end{tabular}

Note: ${ }^{*} \mathrm{p}<0.05$

(24\%) while the basal ganglia lesion had the highest prevalence in the non-pneumonia group (30.9\%) (Table 2).

Cognitive function was evaluated using the MMSE. The MMSE scores were lower in the pneumonia group (22.6 \pm 5.4 vs. $24.7 \pm 4.8$; $\mathrm{p}$-value $=0.032)$, which was statistically significant (Table 3).

\section{Discussion}

This study has compared the cognitive function of ischemic stroke patients with and without pneumonia, resulting that the pneumonia complication is more common in men, office worker, patients with hemiparesis, lower GCS, lower NIHSS, and lesion in parietal lobe. This finding is consistent with a retrospective study showing that ischemic stroke patients are predominantly prevalent in men. ${ }^{9}$ Women have a lower incidence of stroke due to the protective effects of estrogen on cerebral and peripheral vascular disease. Estrogen may increase blood flow, by decreasing vascular reactivity; whereas testosterone has the opposite effect. In addition, genes on the Y-chromosome are partially involved in higher blood pressure and hypertension in men. ${ }^{10}$ Interestingly, a study in Guangzhou, China ${ }^{11}$ has shown that ischemic stroke incidence is higher in patients with a higher education level who are already retired. Higher socioeconomic status is associated with an increased risk of ischemic stroke due to cardiovascular disease and an unhealthy lifestyle. ${ }^{11}$ Furthermore, our study also found that in the group with pneumonia and without pneumonia there were office workers at $44 \%$ and $36.7 \%$, respectively. Office workers experience increased stress from their life and work which might affect blood pressure and cerebral endothelium. However, another study has found that stroke risk is inversely related to education level. ${ }^{12}$

In a previous study in Bandung ${ }^{13}$, hemiparesis is the most common neurologic deficit occurred in stroke-associated pneumonia (SAP) patients, which is consistent with our findings $(87.8 \%)$. It is associated with prolonged immobilization and increased sputum accumulation which predisposes the patient to infection. Hemiparesis, dysarthria, and dysphagia are among the many risk factors that contributed to the SAP incidence. ${ }^{13,14}$ Our study shows that dysphagia is more prevalent in pneumonia group, similar to other studies, showing that dysphagia has increased the risk of pneumonia more than 3-folds among stroke patients. ${ }^{15}$ Dysphagia has impaired the protective reflexes of the laryngeal and swallowing, leading to an increased risk of aspiration..$^{5,15}$ It is therefore important to identify and modify these risk factors in stroke patients. Nil per os status at admission, institutionalized formal dysphagia screening protocol, enteric feeding method, and early mobilization are some of the prophylactic measures against SAP. These should be done to limit the SAP complication that may have a detrimental effect on stroke outcome. ${ }^{14}$

This study has also showed that ischemic lesion mostly occurred in the parietal lobe in the pneumonia group whereas the basal ganglia in the non-pneumonia group. The middle cerebral artery (MCA) is the most common artery involved in stroke and is a major determinant of stroke-associated infection. ${ }^{15,16}$ The lateral surface of the brain and the basal ganglia are supplied by MCA through four segments (M1, M2, M3, M4). Basal ganglia are supplied by M1 and the parietal lobe by M2. ${ }^{16}$ Interestingly, MCA infarction is a strong predictor of cognitive impairment due to its association with working memory tasks $(\mathrm{OR}=2.96) .{ }^{17}$ Basal ganglia connection with prefrontal cortex is associated with cognition and memory function. It also supports habit-like learning and language processing. The parietal cortex contributes to spatial perception and attention. Damage to the basal ganglia or parietal cortex might impair cognitive function. ${ }^{18}$

This study found that the mean age for pneumonia group is lower than nonpneumonia group. Our result is in contrary to a retrospective case-control study, showing that older age is one of many risk factors 
for stroke-associated pneumonia. ${ }^{5}$ Several factors are associated with an increased risk of pneumonia in older age groups, such as immune senescence and an increased risk of aspiration in the elderly. ${ }^{5}$ The findings in our study are in line with a study, showing that decreased level of consciousness and stroke severity predispose stroke patients to develop pneumonia. ${ }^{13}$ Decreased level of consciousness is associated with decreased cough reflex, impaired esophageal sphincter, and swallowing function, thus, increasing the aspiration risk..$^{13}$ Furthermore, our study shows that the pneumonia group has higher NIHSS score. A higher NIHSS score indicates a more severe stroke and is related to a larger infarct size, ${ }^{4,5}$ leading to immunosuppression state making the patients more susceptible to infections. ${ }^{13}$ Higher NIHSS scores are also associated with worse cognitive impairment $(\mathrm{OR}=1.35){ }^{17}$

Our study shows that MMSE scores are lower in the pneumonia group, consistent with a cohort study on cognitive status using the Teng Modified Mini-Mental State (3MS) examination in cardiovascular patients, so cognitive function and pneumonia have a bidirectional relationship. ${ }^{19}$ During an episode of pneumonia, the systemic inflammatory level is increased and the higher levels may accelerate time to dementia caused by neuroinflammation. ${ }^{19}$ Pneumonia is aggravated the ischemic cascade by increasing T Helper 1 directed against central nervous system (CNS) antigens. ${ }^{6}$ Moreover, pneumonia is associated with hypoxia that begins the ischemic stroke cascade, leading to excite toxicity and oxidative damage, resulting in aggravated injury to neuron, glia, and endothelial cells. ${ }^{20}$

This study has several limitations. Of the 427 potential subjects, only 164 fulfilled the inclusion criteria. This might be due to the natural history of the disease since most ischemic stroke patients are admitted in the hospital with decreased consciousness or have aphasia, thus, the MMSE assessment cannot be performed on these patients. The overall findings of this study have shown that higher NIHSS scores are associated with pneumonia, which is known to be a predictor of worse cognitive impairment in stroke patients and is considered a confounding factor. ${ }^{17}$ Additionally, this study do not consider other confounding factors, such as recurrent stroke and vascular risk factors that might interfere with the study outcomes.

In conclusion, cognitive function is more prevalent in ischemic stroke patient having pneumonia compared to non-pneumonia, suggesting this indicates a relationship between cognitive function of ischemic stroke patients and pneumonia. Other factors such as recurrent stroke and vascular risk factors should be considered for future studies to better identify the association of ischemic stroke and pneumonia.

\section{References}

1. Osemene NI. The neurologic complications of ischemic stroke. US Pharm. 2013;38(1):HS1-5

2. Kalaria RN, Akinyemi R, Ihara M. Stroke injury, cognitive impairment and vascular dementia. Biochim Biophys Acta. 2016;1862(5):915-25.

3. 3Sun JH, Tan L, Yu JT. Post-stroke cognitive impairment: Epidemiology, mechanisms and management. Ann Transl Med. 2014;2(8):80.

4. Nam KW, Kwon HM, Lim JS, Lee YS. Leukoaraiosis is associated with pneumonia after acute ischemic stroke. BMC Neurol. 2017;17(1):51.

5. de Castillo LLC, Sumalapao DEP, Pascual JLR. Risk factors for pneumonia in acute stroke patients admitted to the Emergency Department of a Tertiary Government Hospital. Natl J Physiol Pharm Pharmacol. 2017;7(8):855-9.

6. Winklewski PJ, Radkowski M, Demkow U. Cross-talk between the inflammatory response, sympathetic activation and pulmonary infection in the ischemic stroke. J Neuroinflammation. 2014;11:213.

7. Davydow DS, Hough CL, Levine DA, Langa KM, Iwashyna TJ. Functional disability, cognitive impairment, and depression after hospitalization for pneumonia. Am J Med. 2013;126(7):615-24.e5.

8. Bour A, Rasquin S, Boreas A, Limburg M, Verhey F. How predictive is the MMSE for cognitive performance after stroke? J Neurol 2010;257(4):630-7.

9. Habibi-Koolaee M, Shahmoradi L, Kalhori SRN, Ghannadan H, Younesi E. Prevalence of stroke risk factors and their distribution based on stroke subtypes in Gorgan: a retrospective hospitalbased study-2015-2016. Neurol Res Int. 2018;2018:2709654.

10. Haast RAM, Gustafson DR, Kiliaan AJ. Sex differences in stroke. I Cereb Blood Flow Metab. 2012;32(12):2100-7.

11. Wang S, Shen B, Wu M, Chen C, Wang J. Effects of socioeconomic status on risk of 
ischemic stroke: a case-control study in the Guangzhou population. BMC Public Health. 2019;19(1):648.

12. Jackson CA, Sudlow CLM, Mishra GD. Education, sexandriskofstroke:aprospective cohort study in New South Wales, Australia. BMJ Open. 2018;8:e024070.

13. Wandira RD, Amalia L, Fuadi I. Hubungan antara derajat keparahan stroke dengan kejadian stroke-associated pneumonia. Neurona. 2018;35(2):116-20.

14. Hannawi Y, Hannawi B, Rao CPV, Suarez JI, Bershad EM. Stroke-associated pneumonia: major advances and obstacles. Cerebrovasc Dis. 2013;35(5):430-43.

15. Liu DD, Chu SF, Chen C, Yang PF, Chen $\mathrm{NH}$, He X. Research progress in stroke-induced immunodepression syndrome (SIDS) and stroke-associated pneumonia (SAP). Neurochem Int. 2018;114:42-54.

16. Hui C, Tadi P, Patti L. Ischemic Stroke. [Updated 2020 Aug 10]. In: StatPearls
[Internet]. Treasure Island (FL): StatPearls Publishing; 2020 [cited 2020 Dec 5] Available from: https://www.ncbi.nlm.nih. gov/books/NBK499997/

17. Jaillard A, Grand S, Le Bas JF, Hommel M. Predicting cognitive dysfunctioning in nondemented patients early after stroke. Cerebrovasc Dis. 2010;29(5):415-23.

18. Squire L, Berg D,Bloom FE, du Lac S, Ghosh A, Spitzer NC. Fundamental Neuroscience. 3 rd Ed, Cambridge, Massachusetts: Academic Press Elsevier; 2008. p.1277

19. Shah FA, Pike F, Alvarez K, Angus D, Newman AB, Lopez 0, et al. Bidirectional relationship between cognitive function and pneumonia. Am J Respir Crit Care Med. 2013;188(5):586-92.

20. Lakhan SE, Kirchgessner A, Hofer M. Inflammatory mechanisms in ischemic stroke: therapeutic approaches. J Transl Med. 2009;7:97. 